\title{
Processing and Behavior of Fe-Based Metallic Glass Components via Laser-Engineered Net Shaping
}

\author{
B. ZHENG, Y. ZHOU, J.E. SMUGERESKY, and E.J. LAVERNIA
}

In this article, the laser-engineered net shaping (LENS) process is implemented to fabricate netshaped Fe-based Fe-B-Cr-C-Mn-Mo-W-Zr metallic glass (MG) components. The glass-forming ability (GFA), glass transition, crystallization behavior, and mechanical properties of the glassy alloy are analyzed to provide fundamental insights into the underlying physical mechanisms. The microstructures of various LENS-processed component geometries are characterized via scanning electron microscopy (SEM), X-ray diffraction (XRD), differential scanning calorimetry (DSC), and transmission electron microscopy (TEM). The results reveal that the as-processed microstructure consists of nanocrystalline $\alpha$-Fe particles embedded in an amorphous matrix. An amorphous microstructure is observed in deposited layers that are located near the substrate. From a microstructure standpoint, the fraction of crystalline phases increases with the increasing number of deposited layers, effectively resulting in the formation of a functionally graded microstructure with in-situ-precipitated particles in an MG matrix. The microhardness of LENS-processed Fe-based MG components has a high value of $9.52 \mathrm{GPa}$.

DOI: $10.1007 / \mathrm{s} 11661-009-9828-\mathrm{y}$

(C) The Author(s) 2009. This article is published with open access at Springerlink.com

\section{INTRODUCTION}

IN the past decade, a series of new bulk metallic glasses (MGs) with a multicomponent chemistry and high glass-forming ability (GFA) have been developed, including Zr-, Mg-, La-, Pd-, Ti-, and Fe-based alloy systems, ${ }^{[1-4]}$ using various solidification techniques; this has engendered interest in the synthesis and application of bulk MGs. In turn, the properties of these bulk MGs, the strength and anticorrosion properties of which are typically superior to those of their crystalline counterparts, have stimulated researchers to explore techniques to fabricate net-shaped bulk MG components. While some progress has been documented, ${ }^{[5-8]}$ significant breakthroughs have been hindered by the less-thanoptimum GFA and high viscosity that are typically associated with MGs.

Compared with most other MGs, such as $\mathrm{Zr}$ - and Pd-based MGs, the advantages of Fe-based MGs include a much lower material cost and excellent mechanical and physical properties. ${ }^{[8]}$ The major obstacle to forming Fe-based MGs has traditionally been their limited GFA, although some progress has been documented in the literature. ${ }^{[8-11]}$ The high GFA value of these Fe-based MGs has been rationalized on the basis of three empirical rules proposed by Inoue et al.: ${ }^{[12,13]}$ (1) multicomponent alloy systems consisting

B. ZHENG, Postdoctoral Researcher, Y. ZHOU, Associate Researcher, and E.J. LAVERNIA, Provost and Executive Vice Chancellor, are with the Department of Chemical Engineering and Materials Science, University of California, Davis, CA 95616. Contact e-mail: bzheng@ucdavis.edu J.E. SMUGERESKY, Senior Staff Member, is with Sandia National Laboratories, Livermore, CA 94551-0969.

Manuscript submitted August 26, 2008.

Article published online March 31, 2009 of more than three constituent elements, (2) significantly different atomic size ratios, and (3) negative heats of mixing among the constituent elements. It was also reported that the addition of Mo increases the GFA of Fe-based alloys. ${ }^{[11,14]}$ The addition of Mo and W to Fe-based alloys can also enhance their corrosion resistance. ${ }^{[14-16]}$ Moreover, adding $\mathrm{Zr}$ significantly increases the glass transition temperature $T_{g}$, and adding $\mathrm{Mn}$ decreases the liquidus temperature and Curie point. At the same time, increasing the amount of $\mathrm{B}$ can also enhance GFA. ${ }^{[1]}$ In this study, gas-atomized $\mathrm{Fe}_{58} \mathrm{Cr}_{15} \mathrm{Mn}_{2} \mathrm{~B}_{16}$ $\mathrm{C}_{4} \mathrm{Mo}_{2} \mathrm{Si}_{1} \mathrm{~W}_{1} \mathrm{Zr}_{1}$ (at. pet) powder was used for the laser processing of MG components. The other elements (e.g., $\mathrm{Cr}, \mathrm{B}$, and $\mathrm{Si}$ ) enhance thermal stability and provide increased additional solid solution strengthening.

Gas atomization (GA) is a practical and effective approach for producing MG powder. However, the gasatomized MG powder cannot be used directly as a structural component. Accordingly, gas-atomized powder is generally processed through a series of conventional powder metallurgy steps such as degassing, cold isostatic pressing or hot isostatic pressing, and extrusion to form dense bulk materials, which are then machined into final components. In this article, we explore the potential of using the LENS process to form fully dense, three-dimensional (3-D) net-shaped Fe-based MG components through laser deposition. Inspection of the technical literature reveals that the results obtained for the laser glazing ${ }^{[17,18]}$ and laser cladding of $\mathrm{MGs}^{[19-22]}$ have been reported, although these are concerned with surface treatments and coatings. To our knowledge, this work represents the first time that the fabrication of net-shaped bulk MG components has been investigated by laser direct-deposition techniques.

Laser-engineered net shaping (LENS) provides a novel pathway for producing net-shaped bulk MGs. 
The LENS process is a laser-assisted, direct metal manufacturing process originally developed at Sandia National Laboratories (Albuquerque, NM). ${ }^{[23]}$ The LENS process incorporates features from stereolithography and laser cladding, using a computer-aided design file to control the forming process; a 3-D part can be generated point by point, line by line, and layer by layer via additive processing. The LENS process can produce relatively high, localized cooling rates at each deposition point due to the very small size of the melt pool and the conduction of thermal energy into the substrate. ${ }^{[24,25]}$ Therefore, it should be possible to manufacture netshaped MG components using LENS, because the material is deposited as sequential and cumulative layers. In addition, it should be possible to optimize the cooling conditions for MG processing by controlling the processing parameters such as the laser power and travel speed.

The LENS process provides several advantages from an engineering standpoint. The LENS process can be used to generate materials that contain multiple length scales, thereby facilitating the optimization of physical properties. In the case of MGs, it should be possible to fabricate net-shaped components with a high material yield and attractive physical properties. In this article, Fe-based Fe-Cr-Mo-W-C-Mn-Si-Zr-B MG components were deposited via LENS processing. Their microstructure was characterized via scanning electron microscopy (SEM), X-ray diffraction (XRD), differential scanning calorimetry (DSC), and transmission electron microscopy (TEM). The mechanisms thought to be responsible for the observed microstructure, GFA, and mechanical properties are discussed.

\section{EXPERIMENTAL PROCEDURES}

Gas-atomized Fe-based $\mathrm{Fe}_{58} \mathrm{Cr}_{15} \mathrm{Mn}_{2} \mathrm{~B}_{16} \mathrm{C}_{4} \mathrm{Mo}_{2} \mathrm{Si}_{1} \mathrm{~W}_{1} \mathrm{Zr}_{1}$ (at. pct) powder with a size range of 10 to $110 \mu \mathrm{m}$ was selected as the starting material. A hotrolled 304 stainless steel plate with a thickness of $6.35 \mathrm{~mm}$ was used as the substrate. The LENS 750* system used in this

*LENS 750 is a registered trademark of Sandia National Laboratories, Albuquerque, NM.

study was manufactured by Optomec, Inc. (Albuquerque, NM) and consists of a continuous-wave mode Nd:YAG laser operating up to $650 \mathrm{~W}$, a four-nozzle coaxial powder feed system, a controlled-environment glove box, and a motion control system. The nominal laser beam diameter is $6.3 \mathrm{~mm}$ and has a $<0.5-\mathrm{mm}$ diameter circular beam waist at the focal zone. The energy density used in the present article was in the range $2 \times 10^{4}$ to $1 \times 10^{5} \mathrm{~W} / \mathrm{cm}^{2}$.

Three types of sample geometries were fabricated for our study: shells (A, B, and C), solid cubes (D, E, F, and $\mathrm{G})$, and coatings (H, I, J, and $\mathrm{K}$ ), as shown in Figure 1, with different process parameters shown in Table I. In the case of the coating and cubic samples, the area of each layer corresponded to $10 \times 10 \mathrm{~mm}$. The hatch

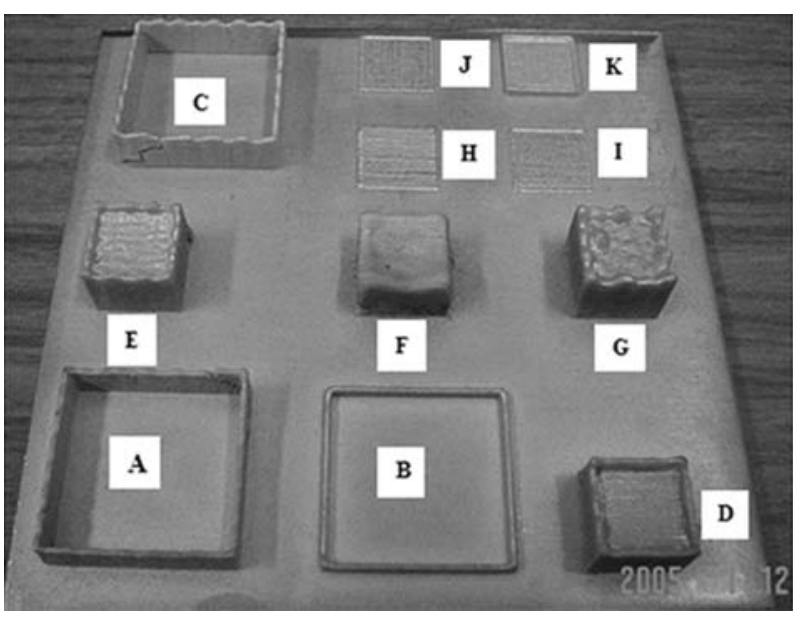

Fig. 1-Laser-deposited Fe-based MG samples using LENS.

space is $0.38 \mathrm{~mm}$ and the layer thickness, $\Delta \mathrm{Z}$, is $0.25 \mathrm{~mm}$. The coating samples were fabricated with different numbers of deposited layers ( 1 for $\mathrm{H}, 2$ for I, 4 for $\mathbf{J}$, and 8 for $\mathrm{K}$ ), to investigate the microstructure evolution during laser deposition. Successive layers were deposited with the hatch lines of two adjacent layers at an angle of $90 \mathrm{deg}$. The entire process was carried out in an Ar environment, to avoid oxidation during deposition. The oxygen level in the glove box was maintained below 10 ppm during deposition.

The as-deposited Fe-based components were sectioned along the center axis and perpendicular to the laser travel trace on the top layer; they were then mounted, ground, and, finally, finely polished using the conventional techniques for metallographic characterization studies. Scanning electron microscopy coupled with energy-dispersive X-ray (EDX) spectrum analysis, XRD with $\mathrm{Cu} K_{\alpha}$ radiation, DSC, and TEM were used for the microstructure characterization and phase analysis of the deposited samples. The XRD scans were performed on the free surfaces of the laser-deposited coatings. microhardness measurements were conducted using a Vickers indenter with Buehler MicroMet 2004 apparatus (Buehler Ltd., Lake Bluff, IL) under a 100-g load on metallographically mounted cross sections of the laser-deposited samples.

\section{RESULTS AND ANALYSIS}

\section{A. Gas-Atomized Powder}

The gas-atomized Fe-based Fe-Cr-Mo-W-C-Mn-SiZr-B alloy powder exhibited a lognormal size distribution. Observations of the powder with SEM secondary electrons revealed that most of the powder is spherical in shape, which is typical for a gas-atomized powder, ${ }^{[26]}$ with variations in the surface morphology, as shown in Figure 2(a). The surface of the smaller powder was generally smoother than that of the larger powder, the associated roughness being attributed to solidification shrinkage. ${ }^{[26,27]}$ Moreover, the surface features were noted to be indicative of the microstructure: smooth in 
Table I. Process Parameters Used for Laser Deposition via LENS

\begin{tabular}{|c|c|c|c|c|c|}
\hline Sample & Number & $\begin{array}{c}\text { Laser Power, } \\
P(\mathrm{~W})\end{array}$ & $\begin{array}{l}\text { Travel Speed, } \\
v(\mathrm{~mm} / \mathrm{s})\end{array}$ & $\begin{array}{c}\text { Power Exposure, } \\
P / v(\mathrm{~J} / \mathrm{mm})\end{array}$ & $\begin{array}{l}\text { Powder Feed } \\
\text { Rate }(\mathrm{g} / \mathrm{min})\end{array}$ \\
\hline \multirow[t]{3}{*}{ Shell } & $\mathrm{A}$ & 180 & 12.7 & 14.17 & 10 \\
\hline & B & 280 & 8.47 & 33.06 & 6 \\
\hline & $\mathrm{C}$ & 280 & 12.7 & 22.05 & 10 \\
\hline \multirow[t]{4}{*}{ Cubic bulk } & $\mathrm{D}$ & 180 & 12.7 & 14.17 & 10 \\
\hline & $\mathrm{E}$ & 180 & 8.47 & 21.25 & 10 \\
\hline & $\mathrm{F}$ & 296 & 8.47 & 34.83 & 10 \\
\hline & $\mathrm{G}$ & 180 & 4.23 & 42.55 & 10 \\
\hline Coating & $\mathrm{S}, \mathrm{T}, \mathrm{U}$, and $\mathrm{V}$ & 180 & 12.7 & 14.17 & 10 \\
\hline
\end{tabular}

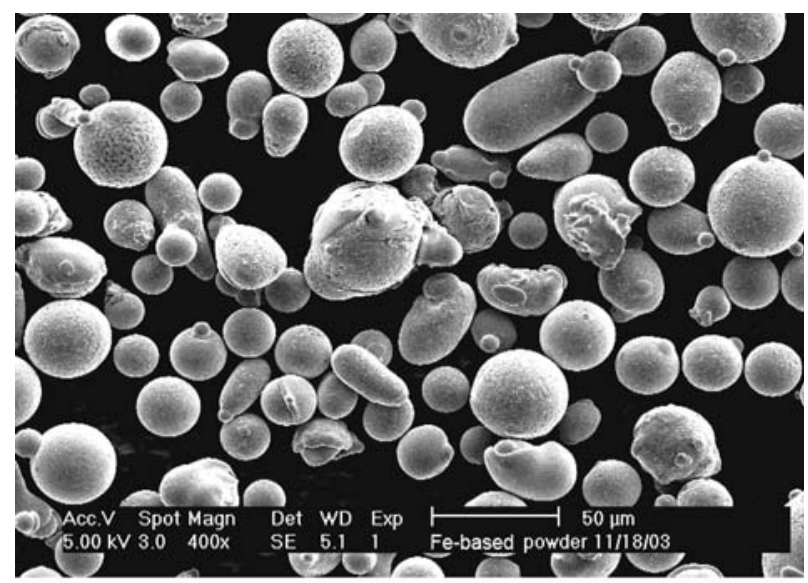

(a)

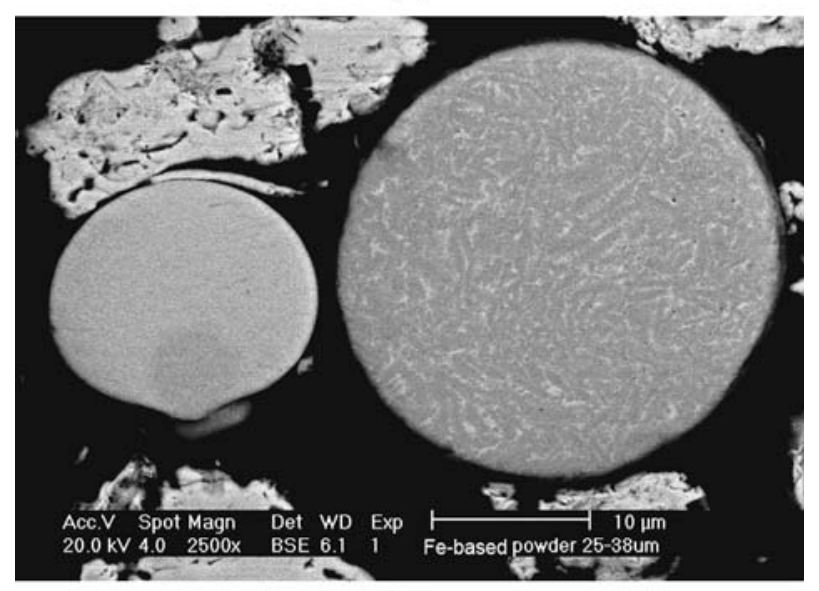

(b)

Fig. 2-SEM micrographs of gas-atomized Fe-based alloy powder: (a) powder morphology and (b) powder cross section.

the case of the amorphous powder and rough in the case of the crystalline powder.

The cross-sectional microstructures of the different powder sizes were studied using SEM backscattered electrons (BSEs) and revealed the typical microstructure shown in Figure 2(b). The powder $<20 \mu \mathrm{m}$ revealed a featureless microstructure, indicating that no crystallization had occurred during GA. The featureless powder was considered to be amorphous. The extent of crystallization was noted to increase with the powder size, consistent with published results. ${ }^{[28-30]}$ It is important to note that, although a starting powder with a wide particle size range (from 10 to $110 \mu \mathrm{m}$ ) and different initial microstructures (from amorphous to crystalline) was used in this work, all the particles will be completely melted and resolidified during LENS deposition. Therefore, the influence of the initial microstructure of the starting powder on the microstructure and properties of the laser-deposited layers was deemed to fall outside of the scope of the present study. The primary considerations used to select the starting powder were morphology (i.e., spherical), size $(<150 \mu \mathrm{m})$, GFA, and laser energy absorption. For the irradiation with Nd:YAG laser (wavelength $1.06 \mu \mathrm{m})$, the absorptivity of $\mathrm{Al}(0.06$ to $0.2)$ and $\mathrm{Cu}(0.04$ to 0.3$)$ alloys is much lower than that of $\mathrm{Fe}$ alloys $(0.25$ to 0.35$){ }^{[31]}$

\section{B. Microstructure of LENS-Deposited Samples}

The material initially deposited on the cold substrate during LENS experiences a high quenching effect; it is in this region that an amorphous microstructure is likely to be observed. The magnitude of the cooling rate during the first-layer deposition can be estimated to be $10^{3}$ to $10^{4} \mathrm{~K} / \mathrm{s}$ on the basis of numerical simulations. ${ }^{[32,33]}$ Figure 3 shows a micrograph, imaged with SEM BSEs, of an initially deposited layer obtained with a laser output power of $280 \mathrm{~W}$ and a travel speed of $12.7 \mathrm{~mm} / \mathrm{s}$. The features of the melt pool shape and the overlap between subsequent deposited lines are clearly visible. The microstructure is featureless, which suggests that the first layer is amorphous. This indicates that the cooling rate experienced by the deposited materials was high enough to form an amorphous microstructure, consistent with that reported for these Fe-based MGs. ${ }^{[19,34]}$ The top surface contained some partly unmelted particles that retained the original partially amorphous structure of the powder, as shown in Figure 3(a). These particles will be completely melted and resolidified during the subsequent layer deposition, because the surface of the previously deposited layer is always partially remelted by the laser beam during deposition. Only the surface of the final deposited layer contains partly unmelted particles. The presence of light-colored phases in the second layer suggests that melting and resolidification occurred in this region presumably under a low cooling rate, e.g., approximately $10^{2}$ to $10^{3} \mathrm{~K} / \mathrm{s}^{[33,35]}$

In order to investigate any possible reactions of the deposited layer with the substrate, an identical powder 


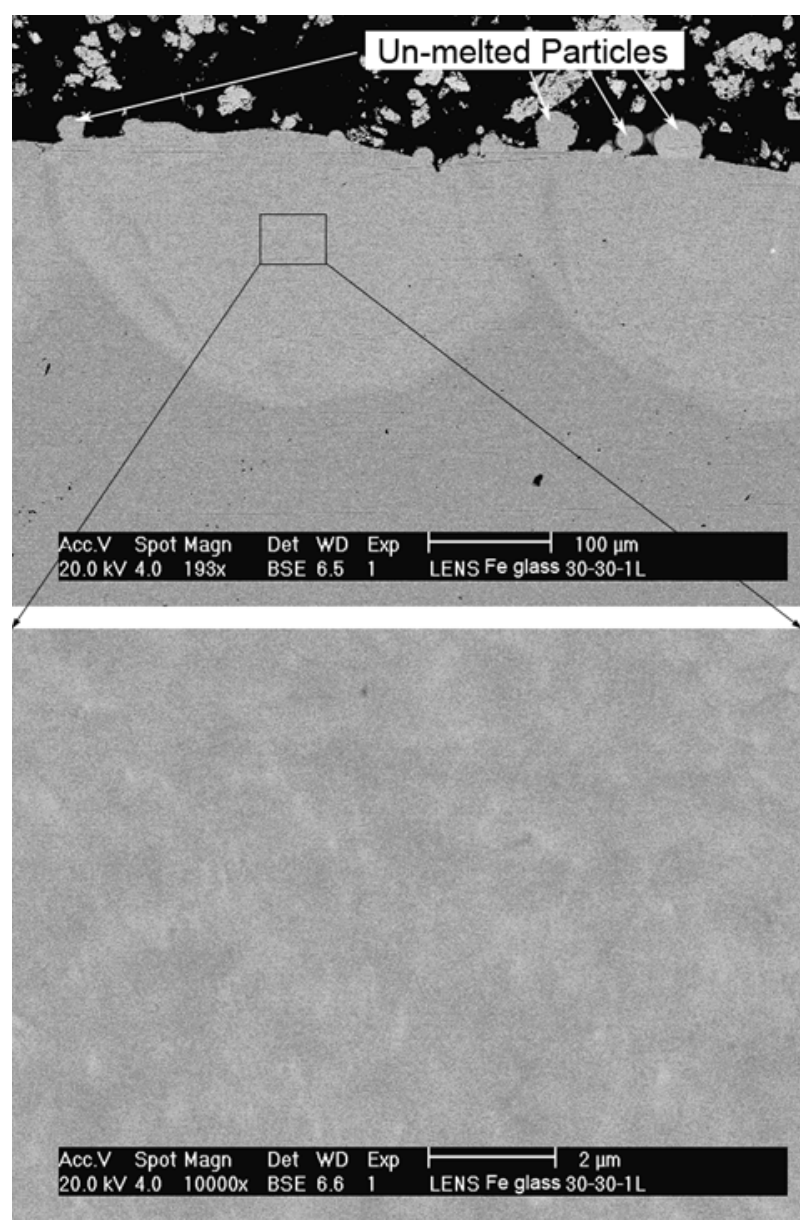

Fig. 3-SEM (BSE) micrographs of first layer of laser-deposited Fe-based MG coating via LENS.

was deposited on an amorphous substrate using the same parameters as were used for deposition on the 304 SS substrate. Similar to the case of a crystalline substrate, laser deposition on the amorphous substrate made of Fe-based Fe-Cr-Mo-W-C-Mn-Si-Zr-B thick amorphous coatings $(400 \mu \mathrm{m})$ on a 304 SS plate via high-velocity oxygen fuel thermal spraying ${ }^{[36]}$ also resulted in overlapping, amorphous melt zones. The absence of microstructural features in these zones indicates that the region is initially melted by the laser beam and then resolidified as glass, regardless of the nature of the underlying substrate. The absence of significant dilution or reaction products at the interface of the deposited Fe glass and the 304 SS substrate is attributed to the high solidification rates associated with LENS processing, which suppresses the diffusioncontrolled phase transformations of crystallization such that solute trapping will occur and large segregation no longer operates.

As the thickness of the deposited material increases, the microstructure coarsens and precipitation becomes increasingly evident, particularly at the boundary of the melt pool region, which, for purposes of this discussion, may be described as a heat-affected zone (HAZ). The thickness and morphology of the HAZ strongly depends on the LENS processing parameters. This trend is

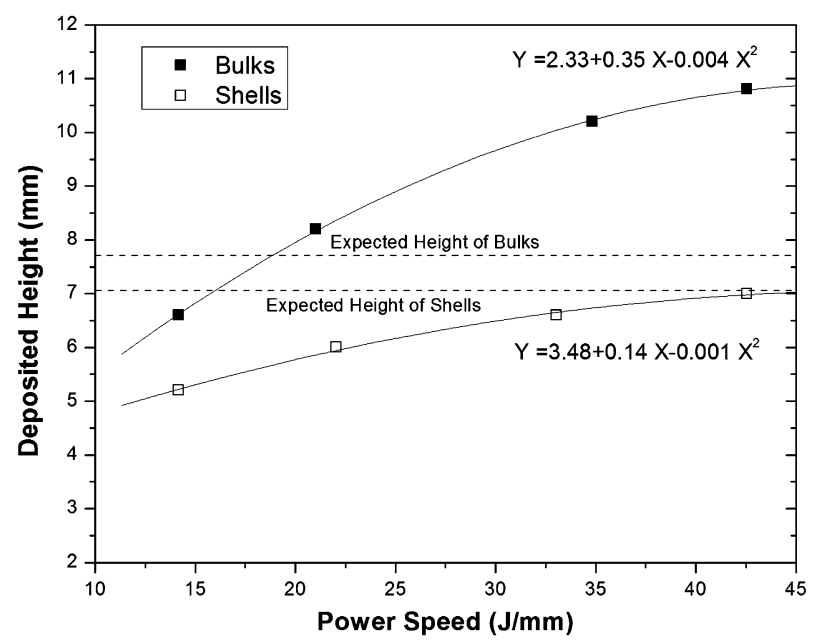

Fig. 4-Variation on build-up height of materials with laser power exposure.

consistent with the decrease in the cooling rate that accompanies an increase in the number of deposited layers.

In the case of the LENS-deposited samples with cubic and shell geometries, the macroscale variables that influence deposition are a buildup of height and melt depth into the previous layer. To ascertain the results of these samples, metallographic cross sections of the deposited materials were made. The height buildup was measured from the substrate surface to the upper surface of the deposited material. Similarly, the melt depth was taken to be the depth of the region in which dissolution is evident. The measured melt depth tends to increase from a minimum value of $0.05 \mathrm{~mm}$ to a maximum value of $0.25 \mathrm{~mm}$ with increasing laser power and decreasing laser travel speed. When the laser travel speed is low, there is time for powder accumulation, and slightly more penetration occurs. In terms of geometrical considerations, the laser power and travel speed also played a significant role in the accumulation and remelting processes. The experimental results suggest that there was a strong correlation between the height buildup of the materials and a ratio defined hereafter as the laser power exposure (laser powder/laser travel speed). Figure 4 shows the variation in the height buildup with the laser power exposure. The overall thickness of the bulk cubic and shell samples increases with increasing laser power exposure. The relationship between the height buildup $H$ and the processing parameter $P / v$ was derived using curve-fitting techniques; it is given as

$H=2.33+0.35(P / v)-0.004(P / v)^{2}$ for bulk geometries

$H=3.48+0.14(P / v)-0.001(P / v)^{2}$ for shell geometries

where $P$ is the laser output power and $v$ is the laser travel speed. The thickness of the bulk geometry is larger than 


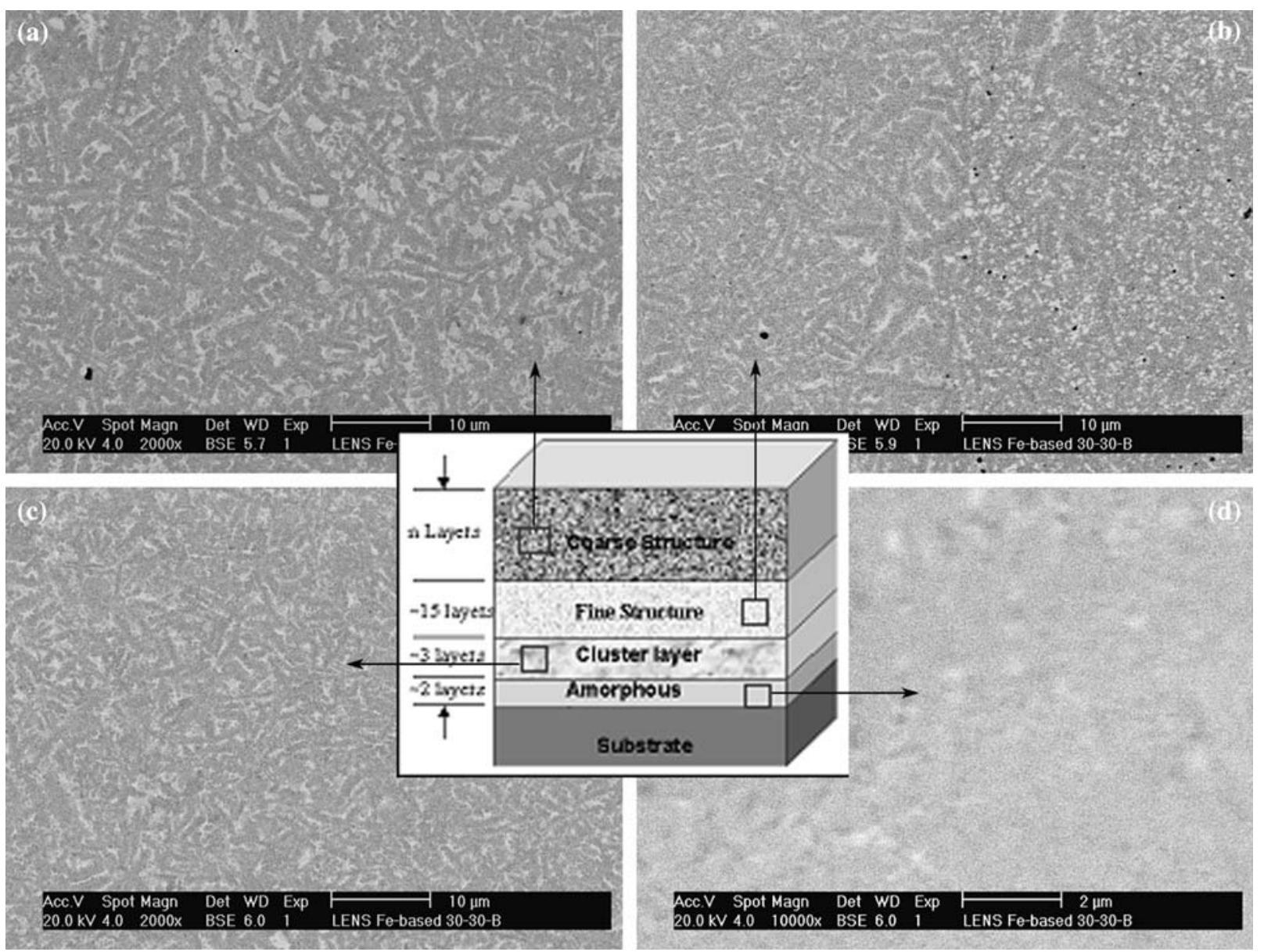

Fig. 5-SEM (BSE) micrographs of laser-deposited Fe-based MG cube.

that of the shells and can be attributed to the fact that there is overlap between the deposition lines during the fabrication of the former, whereas there is none during the deposition of the latter.

The typical microstructure of laser-deposited bulk cubic geometry is shown in Figure 5. This sample was produced with a laser power of $280 \mathrm{~W}$ and a travel speed of $12.7 \mathrm{~mm} / \mathrm{s}$. It is evident that the microstructure from the top layers is much coarser than that present in the bottom layers. The results from this sample confirm that the temperature of the deposited materials increases with thickness during laser deposition, and that the cooling rate decreases with increasing distance from the substrate. In addition, the heat generated by the subsequently deposited layers can promote crystallization of the preceding layers. Approximately two layers revealed a featureless microstructure, which was considered to be amorphous.

Figure 6 shows the microstructure evolution in the laser-deposited Fe-based MG shell component processed with a laser output power of $280 \mathrm{~W}$ and a travel speed of $12.7 \mathrm{~mm} / \mathrm{s}$. As one moves from the bottom to the top layers, the microstructure varies from amorphous to an MG matrix composite, effectively forming a functionally graded microstructure. It is interesting to note that, if one compares the microstructure of the shell sample to that of the corresponding location in the cubic sample, it is shown that the former is coarser than the latter (Figure 5). This difference is attributed to the higher thermal conductivity of the bulk metal (cubic sample) as compared to the heat dissipated via convection into the environment (shell sample). In the case of a given set of process parameters, the microstructural evolution during LENS is primarily influenced by the conduction of thermal energy through the deposited material into the substrate, which effectively behaves as a heat sink. Convective and radiative losses into the environment appear to have only a limited effect on the microstructural evolution. An additional factor that contributes to the observed difference in microstructure between the shell and cubic samples is the fact that the time interval between two sequential layers is shorter for the shell sample than for the cubic sample. According to the experimental and numerical simulation results, ${ }^{[24,25,33]}$ the temperature at the end of each deposition cycle increases with a decreasing interval time, which corresponds to a decrease in the cooling rate during deposition. In addition, the accumulation of thermal energy that is likely to develop during the deposition of multiple layers is also likely to promote crystallization of the microstructure. 


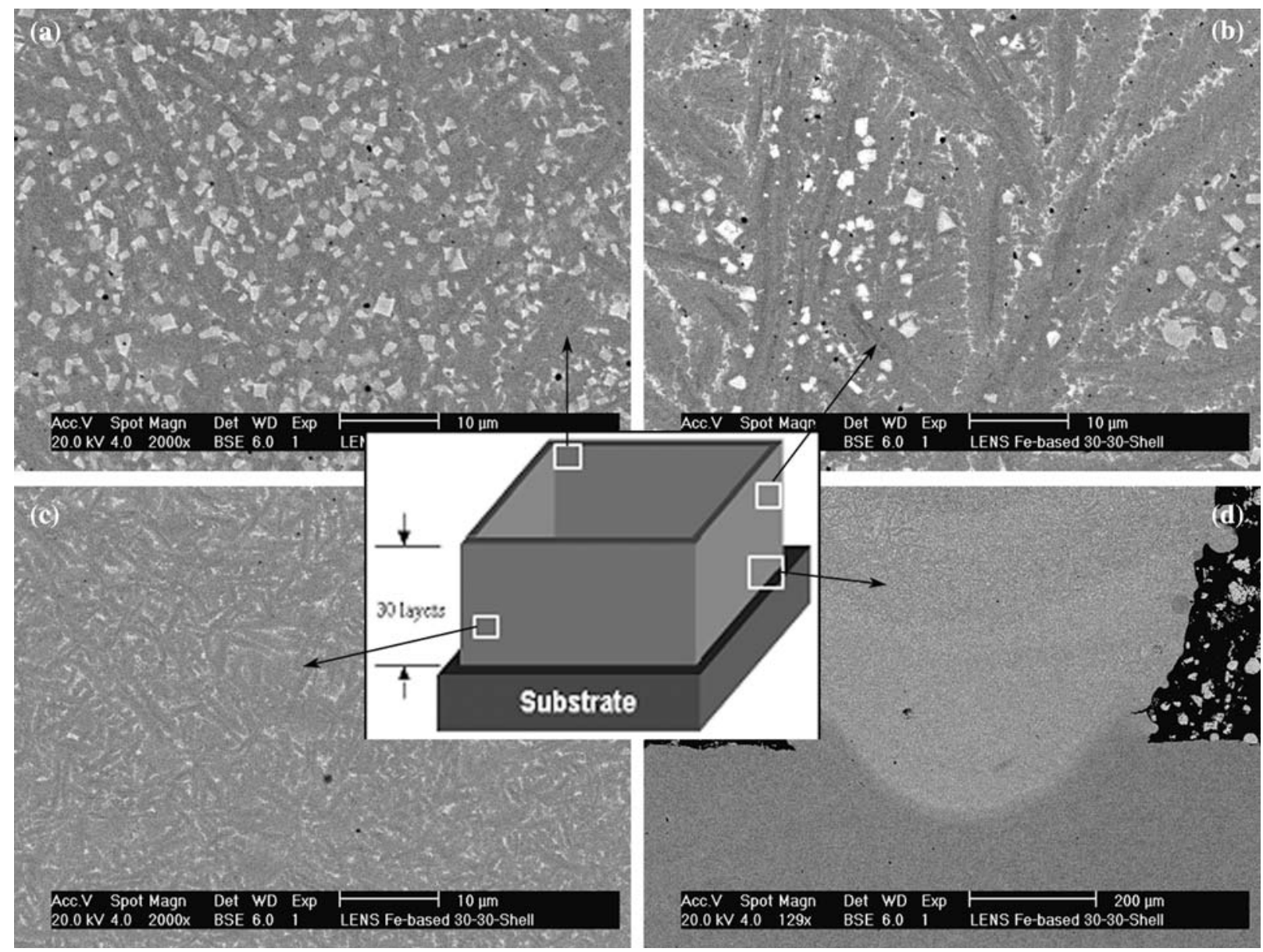

Fig. 6-SEM (BSE) micrographs of laser-deposited MG shell component.

The energy-dispersive analysis line-scanning results (Figure 7) show that the precipitated white phase contained a higher concentration of $\mathrm{Cr}, \mathrm{W}$, and $\mathrm{Mo}$ relative to the matrix. During the initial stages of phase precipitation, the boundary between the precipitated phases and the matrix is not well defined as the component atoms begin to segregate and cluster. The phase morphology of the precipitated phases becomes as well defined as in the upper regions of the samples, because extensive precipitation has been facilitated by a low cooling rate.

Figure 8 shows the XRD patterns obtained from the top surface of laser-deposited Fe-based MG coatings with different numbers of deposited layers. The XRD curve of the deposited first layer presents a diffuse broad halo peak at around $2 \theta=44.4 \mathrm{deg}$, illustrating the amorphous nature of the specimen. The broad diffraction peak becomes less obvious with increasing numbers of deposited layers. The crystalline peaks appeared superimposed on a broad diffraction peak for all the coating samples, which is similar to the pattern obtained from the gas-atomized powder. Even though the SEM BSE images of the first-layer sample revealed a featureless microstructure within a molten pool region, some crystalline peaks still appeared on the corresponding XRD curve. In fact, these peaks were attributed to the precipitated crystalline in the HAZ and partially melted powder, which retained the original partially amorphous structure. Three broad peaks at around 44.4, 64.5, and $81.7 \mathrm{deg}$, corresponding to the reflections of the nanocrystalline $\alpha$-Fe phase, can be observed together with a halo peak, which confirms the presence of precipitated nanocrystalline $\alpha$-Fe embedded in an amorphous matrix. The other phases presented are likely to be $\gamma$-Fe, tetragonal $\mathrm{Fe}_{2} \mathrm{~B}$, cubic $\mathrm{Cr}_{23} \mathrm{C}_{6}$, and other, unidentified phases.

The microstructure of laser-deposited MG samples was also observed using TEM. Although the initial deposited layers appear featureless during observation with SEM, as shown in Figures 3, 5, and 6, some nanocrystallites of $\alpha-\mathrm{Fe}$ with a size of 5 to $20 \mathrm{~nm}$, embedded in an amorphous matrix, were observed via TEM. This is illustrated in Figure 9(a), in which the bright-field image and its corresponding diffraction pattern reveal the presence of $\alpha-\mathrm{Fe}$ diffraction rings. The increased deposition thickness resulted in additional precipitation, as illustrated in Figure 9(b).

The TEM results also confirmed the observation of an increased degree of crystallinity with the increased deposition thickness. This phenomenon is illustrated in Figure 9(b), which shows a bright-field image and its corresponding diffraction pattern from an upper layer. 

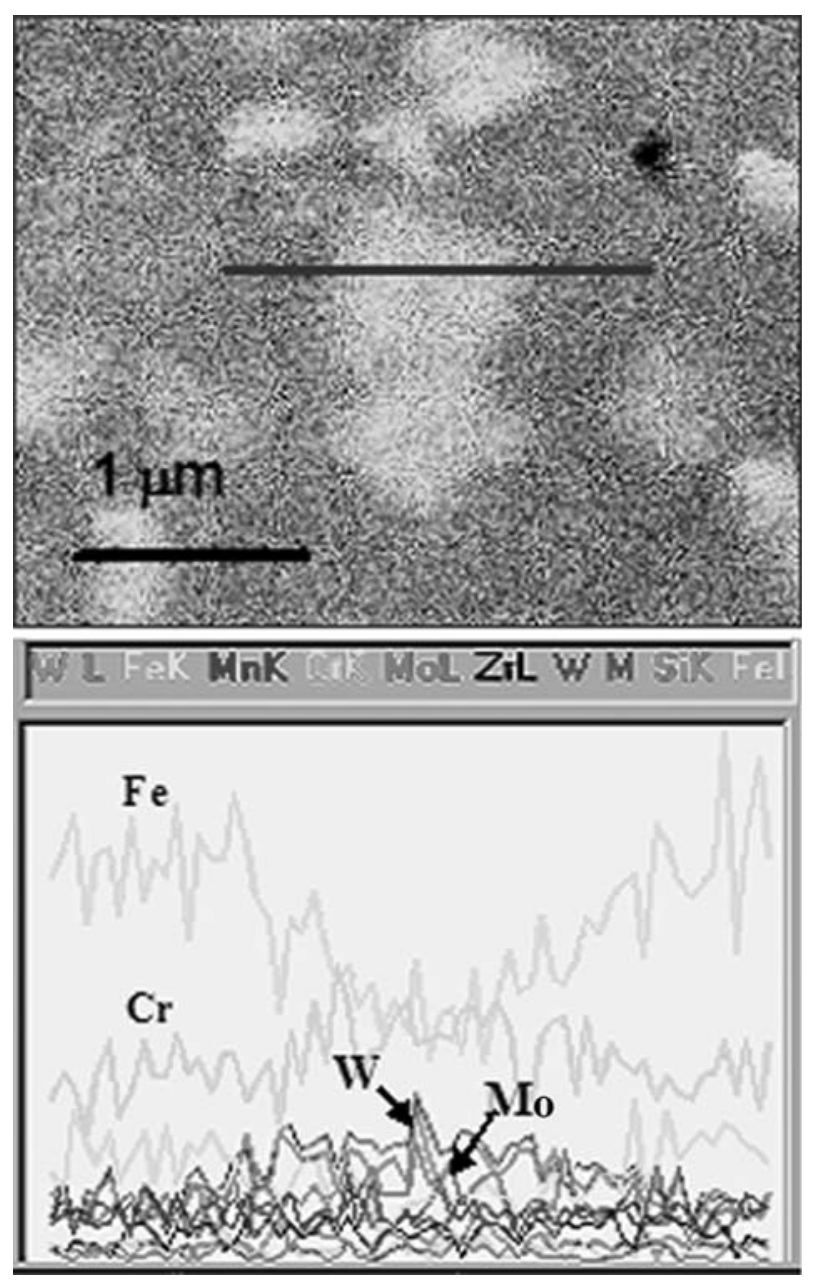

Fig. 7-Element distribution around white phase with EDX line scanning.

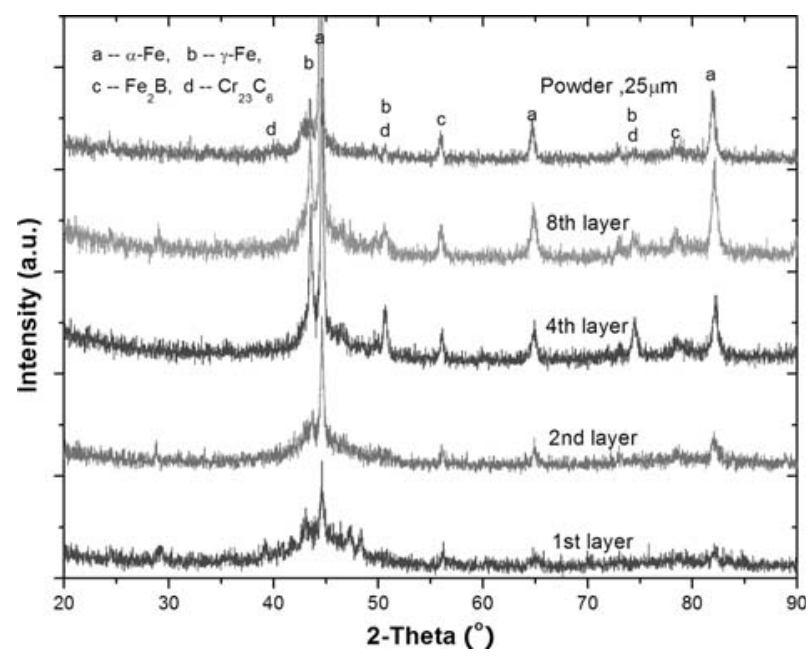

Fig. 8-XRD patterns of laser-deposited Fe-based MG coatings with different numbers of layers.

The size of the precipitated crystals that are evident in this figure is approximately $100 \mathrm{~nm}$. In all of these cases, a primary crystallization reaction occurs and

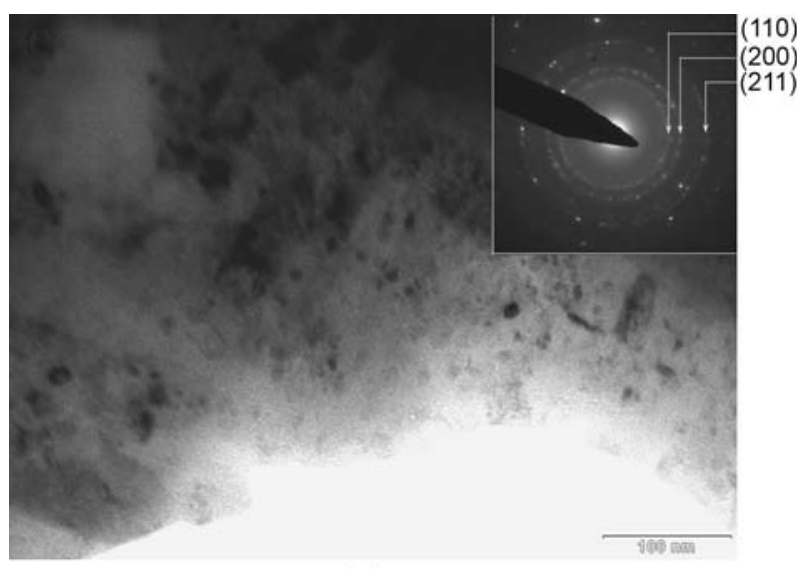

(a)

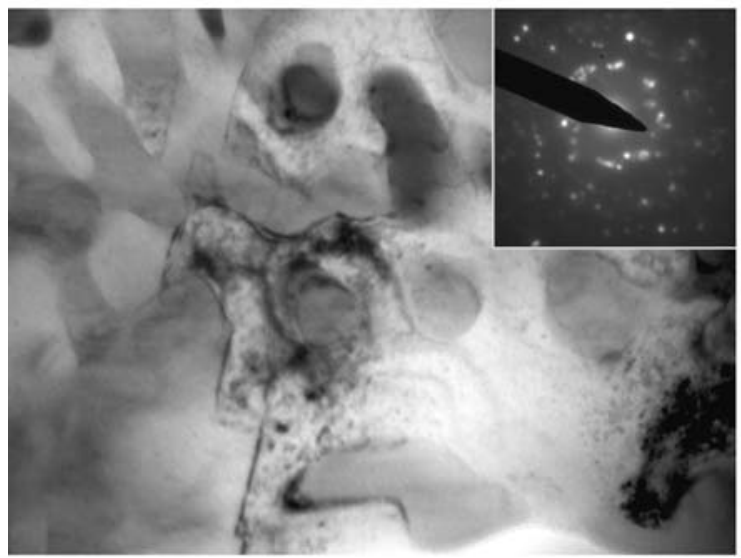

(b)

Fig. 9-TEM micrograph and its diffraction pattern of $(a)$ first layer of laser-deposited Fe-based MG and $(b)$ coarse microstructure of laser-deposited Fe-based MG.

starts with the formation of nanocrystalline $\alpha-\mathrm{Fe}$ grains. Further growth of these particles and the appearance of new grains ensued as additional layers were deposited.

Figure 10 shows the DSC trace curve of the first layer of the laser-deposited Fe-based MG coating, as determined with a heating rate of $20{ }^{\circ} \mathrm{C} / \mathrm{min}$. With increasing temperature, the curve shows an amorphous transition, followed by the appearance of a supercooled liquid region and crystallization. The glass transition temperature $T_{g}$ corresponds to the temperature at which the curvature of the endothermic reaction on the DSC curve is maximum, while the crystallization onset temperature $T_{x}$ is defined as the temperature at which the tangential lines between the supercooled liquid and the exothermic peak intersect each other. The temperature interval of the supercooled liquid region $\Delta T_{x}$, defined by the difference between the glass transition temperature and the onset temperature of crystallization, $\Delta T_{\mathrm{x}}=T_{x}-T_{g}$, is as large as $110{ }^{\circ} \mathrm{C}$. In addition, the $T_{g} / T_{m}$ value is 0.37 . The large supercooled liquid region implies a high thermal stability of the supercooled liquid against crystallization. Generally, the GFA increases with increasing $\Delta T_{\mathrm{x}}$ and $T_{g} / T_{m} \cdot{ }^{[3,37]}$ 


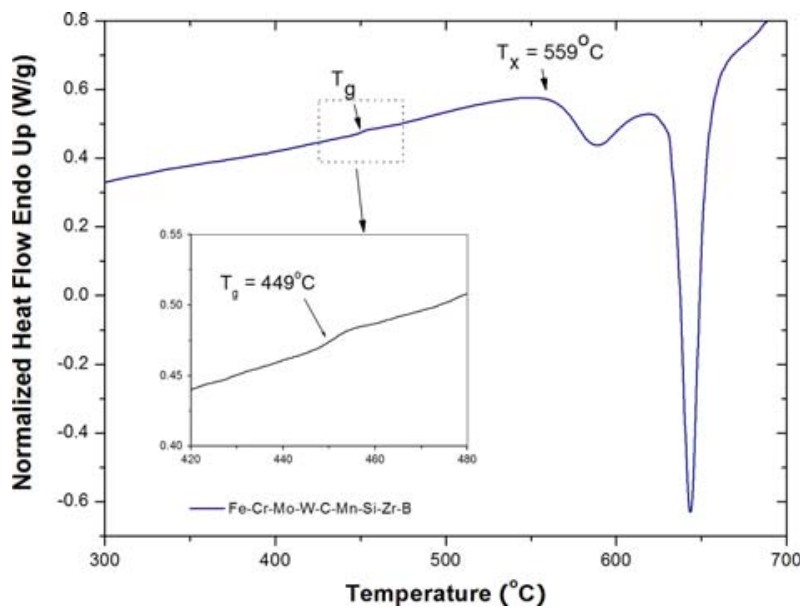

Fig. 10-DSC curve of the first layer of laser-deposited Fe-based MG.

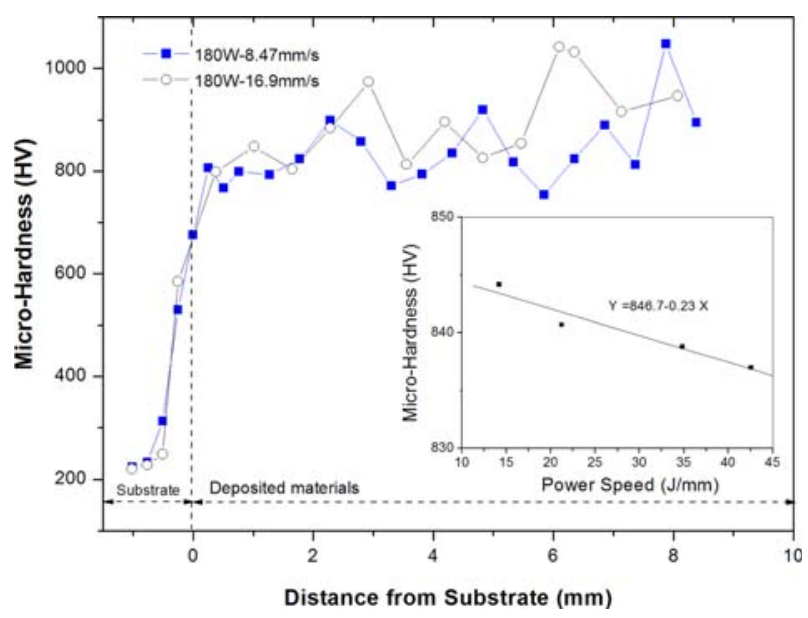

Fig. 11-Variation in microhardness at the center of the bulk sample with distance from substrate $(\mathrm{Z}$ direction) and laser power exposure.

\section{Mechanical Properties}

The microhardness of the LENS-deposited Fe-based Fe-Cr-Mo-W-C-Mn-Si-Zr-B MG component was measured using a 100-g load with a Vickers microhardness tester; the results are summarized in Figure 11. The results show the variation in microhardness at the center of the cubic sample as a function of distance from the substrate surface in the height buildup direction. The variations in microhardness of the cubic MG sample in the transverse direction, as well as in the center of a shell sample, as a function of the distance from the substrate surface were also measured. No significant variations in microhardness were observed in the cases of the transverse measurements for the cubic sample and the center measurements for the shell sample, despite obvious differences in the degree of devitrification. The variation in microhardness values obtained from the bottom region is significantly smaller than that of the upper region, as a result of the predominantly amorphous structure present near the bottom region.
The variations in microhardness summarized in Figure 11 can be attributed to the extent of precipitation throughout the deposited material. It is also interesting to note that the microhardness of the deposited materials was slightly higher for the higher laser travel speed, which corresponds to a decrease in the interaction time of the laser materials and the associated high cooling rate during deposition. The microhardness of laser-deposited Fe-based MG materials is approximately $900 \mathrm{HV}$ $(9.5 \mathrm{GPa})$, with a corresponding tensile strength $(\sim 1 / 3$ hardness) of approximately $3.1 \mathrm{GPa}$, which is significantly higher than the values for conventional steels, e.g., 276 to $1882 \mathrm{MPa}$ for carbon steels, 758 to $1882 \mathrm{MPa}$ for alloy steels, 515 to $827 \mathrm{MPa}$ for stainless steels, and 640 to $2000 \mathrm{MPa}$ for tool steels. ${ }^{[38]}$ The variation in the average microhardness of the LENSdeposited bulk MG samples with laser exposure is also shown in the inset of Figure 11. It was found that the average microhardness decreases almost linearly with increasing laser exposure (laser power/laser travel speed), which corresponds to a decreasing cooling rate during deposition. The relationship between the average microhardness $M H$ and the laser exposure $P / v$ was derived by curve fitting, and is given as

$$
M H=846.7-0.23(P / v)
$$

where $P$ is the laser output power and $v$ is the laser travel speed.

\section{DISCUSSION}

The microstructure evolution of laser-deposited Fe-based Fe-Cr-Mo-W-C-Mn-Si-Zr-B MG materials during LENS processing is complex, because it not only depends on the thermal history but it is also a function of the alloy system. Due to the layer-additive nature of the LENS process, the thermal behavior associated with the LENS process involves numerous reheating cycles. Thus, the assessment of the microstructure evolution necessitates an understanding of the response of the alloy to these cycles. As shown in previous numerical and experimental studies, ${ }^{[25,32,33]}$ the thermal behavior associated with the LENS process involves a series of wave-shaped thermal cycles. Each peak represents a laser heating event as the laser beam passes over a layer and effectively reheats layers that were deposited previously. The thermal excursions dampen out when the laser energy source moves away during the deposition of subsequent layers. After reaching an initial peak temperature, the heat is quickly conducted away for the first layer, which experiences rapid heating and cooling, nonequilibrium conditions. Because of rapid heat loss through the substrate during initial deposition, this initial thermal transient results in a high cooling rate $\left(10^{3}\right.$ to $\left.10^{4} \mathrm{~K} / \mathrm{s}^{[33,35]}\right)$ during solidification and can produce a glassy microstructure in the case of glassforming alloys, as shown in Figures 3 and 9(a). However, each subsequent pass reheats the previously deposited layers such that after several layers are deposited, the initial deposited layers continue to 
experience thermal excursions. When the reheating temperature is higher than the crystallization onset temperature $T_{x}$, new phases will precipitate, implying MG devitrification. However, the time it takes a deposited upper layer to reheat a lower layer to a temperature above $T_{x}$ is very short $(<0.05$ seconds) for each deposition sequence, and the thermal energy is rapidly dissipated into the lower layers. Our microstructural analysis shows that a heating time as short as this was not sufficient to fully crystallize the lower layers.

The accumulation of thermal energy during component fabrication is likely to promote devitrification. The accumulation of thermal energy at the end of each cycle causes the temperature to be somewhat higher than that at the end of the previous cycle. Accordingly, the temperature increases monotonically with the increasing deposited material thickness, as confirmed via numerical and experimental results. ${ }^{[24,33]}$ It then follows that the cooling rate decreases with the deposition thickness, consistent with the observation that the microstructure coarsens from the bottom to the top layers, as illustrated in Figures 5, 6, and 9(b). Only when the deposited sample attains a sufficient thickness will the temperature of a lower layer exceed $T_{x}$ and complete crystallization occur. From our modeling results, ${ }^{[33]}$ the stable temperature for a 25.4- $\mathrm{mm}$ distance from the substrate can reach $400{ }^{\circ} \mathrm{C}$, when the overall deposited material is approximately $50.8 \mathrm{~mm}$. This complex thermal cycling will influence not only the microstructure but also residual stresses as the material is tempered or aged.

There are two key parameters of the LENS process, laser output power and travel speed, that directly determine the local thermal conditions (temperature and cooling rate) and therefore control the resulting microstructure of deposited MGs. These two parameters can be combined into the energy intensity of a laser beam, which can be determined by dividing the power of the beam by the spot area:

$$
I=\frac{P}{v d}
$$

where $P$ is laser output power, $v$ is laser travel speed, and $d$ is the laser beam spot size in diameter on the substrate. For a given laser power, increasing the laser spot size and scanning speed will decrease the intensity, while a high laser power will increase the intensity. This thermal energy input melts the injected powder and the surface layer of the substrate; it also heats the underlying material. Both the depth of the melt zone and the thickness of the HAZ increase with increasing heat input. A fast laser-scanning speed leads to a short interaction time between the laser beam and the material, which can also promote high quenching and lead to the higher strength of the deposited Fe-based MGs, as shown in Figure 11. The process parameters of laser output power, travel speed, and initial temperature of the substrate have significant influences on the thermal history of the deposited materials; the cooling process can be controlled by changing these variables. The present results suggest that a combination of a laser power of $150 \mathrm{~W}$ and a travel speed of $12.7 \mathrm{~mm} / \mathrm{s}$ with a powder feed rate of $10 \mathrm{~g} / \mathrm{min}$ appears to be close to ideal for forming a melt pool necessary for powder incorporation without causing much crystallization in the initial deposition.

The behavior of MGs synthesized via LENS laser deposition depends on both the process parameters and the alloy composition. The Fe-based Fe-Cr-Mo-W-C$\mathrm{Mn}-\mathrm{Si}-\mathrm{Zr}-\mathrm{B}$ alloy used in the present article has a limited GFA, as is evidenced by the microstructure of both the gas-atomized and LENS-deposited samples (Figures 2 and 3 and 4 through 8). The basis of the present alloy is the Fe-Mn-Cr-B system, ${ }^{[2,9-11]}$ which satisfies the three empirical rules. ${ }^{[12,13]}$ The addition of Mo, W, Zr, C, and Si can cause an increase in the atomic size difference and the generation of new atomic pairs with various negative heats of mixing. For a multicomponent MG consisting of $n$ constituent elements, the criterion of the ratio of the atomic size difference $\lambda_{n}$ has been used to evaluate the optimum solute concentration for high a GFA; ${ }^{[39]} \lambda_{n}$ is defined as

$$
\lambda_{n}=\sum_{B=1}^{n-1}\left|\left(\frac{r_{B}}{r_{A}}\right)^{3}-1\right| \cdot C_{B}
$$

where $r_{A}$ and $r_{B}$ are the solvent and solute atom radius, respectively, and $C_{B}$ is the solute concentration (at. pct) of element B. It is found that the values of $\lambda_{n}$ of bulk MG formers with a great GFA in Zr-, Pd-, Mg-, Nd- and Fe-based systems are approximately constant of 0.18 . The $\lambda_{n}$ is 0.21 for the composition corresponding to the $\mathrm{Fe}_{58} \mathrm{Cr}_{15} \mathrm{Mn}_{2} \mathrm{~B}_{16} \mathrm{C}_{4} \mathrm{Mo}_{2} \mathrm{Si}_{1} \mathrm{~W}_{1} \mathrm{Zr}_{1}$ (at. pct) alloy. The large atomic size difference in the multicomponent system can result in the highly dense and randomly packed structure of $\mathrm{MG}$ alloys. The nucleation and growth of the crystalline phase may be suppressed in the supercooled liquid by inhibiting the long-distance diffusion and increasing the melt viscosity. It is also very difficult for the multiple elements in the alloy to simultaneously satisfy the composition and structural requirements of the crystalline compounds. Minor alloying additions are frequently added to MGs, to improve GFA and effectively "tune" the mechanical and physical properties of bulk MGs. ${ }^{[40]}$ The addition of metalloid elements such as $\mathrm{B}, \mathrm{C}$, and $\mathrm{Si}$ has a significant effect on the GFA, thermal stability, and properties of Fe-based MG-forming alloys. Proper additions of small atomic-sized elements such as B, $\mathrm{C}$, and $\mathrm{Si}$ can tighten the alloy structure and stabilize the alloy against crystallization. Minor additions of $\mathrm{Zr}$ enable this alloy to behave as a liquidlike structure at low temperatures and to remain amorphous as it solidifies, due to the strong affinity $\mathrm{Zr}$ has for $\mathrm{O}$. However, the as-received alloy used in the present investigation shows a limited GFA. Recently, Fe-based alloys with a high GFA have been developed (e.g., Fe-Cr-Mo-Y-C-B) and should be of interest for LENS studies, because results show that a fully amorphous structure in the gas-atomized power size range 40 to $150 \mu \mathrm{m}$ were obtained. ${ }^{[41]}$

The observed Fe-based Fe-Cr-Mo-W-C-Mn-Si-Zr-B amorphous phase in the laser-deposited samples can 
be attributed to the rapid solidification that occurs during rapid cooling as the thermal energy is transferred into the substrate. In this case, the nucleation and growth of crystallites are hindered by the local rapid drop in temperature. Beyond the initial layers, however, the temperature of the deposited material constantly increases as additional layers are added, which results in a decrease in the cooling rate and, hence devitrification, as shown in Figures 5 and 6. According to the experimental and modeling results, ${ }^{[25,33]}$ the temperature could increase to a value of approximately $150{ }^{\circ} \mathrm{C}$ at the end of the secondlayer deposition, and the cooling rate would decrease to approximately $10^{-2} \mathrm{~K} / \mathrm{s}$ for the third-layer deposition. This indicates that a constant cooling rate of at least $>10^{-2} \mathrm{~K} / \mathrm{s}$ is required for depositing this type of Fe-based MGs via the LENS process, based on the absence of crystallization.

The inherent variation in the cooling rate during LENS processing can lead to the formation of graded materials with an in-situ-formed MG matrix composite, as shown in Figures 5 and 6. It is interesting to note that this type of microstructure has been reported to be beneficial for strength and fracture toughness. ${ }^{[7,42]}$ In these microstructures, fracture may be suppressed by limiting strain localization by the precipitated reinforcing phase. The precipitated phases can also help distribute both shear bands and microcracks, limit shear band extension, suppress shear band opening, and avoid crack development.

These preliminary research results on LENS-deposited MG components indicate their flexibility in processing novel materials, even those in which the thermal and solidification conditions must meet certain conditions. The cracks that were evident in the deposited MGs, as shown in shell sample $\mathrm{C}$ in Figure 1, are attributable to the lack of ductility of MGs and to residual stress development, which is an intrinsic outcome of incremental deposition processes such as LENS. $^{[43]}$

\section{SUMMARY}

The microstructure of the gas-atomized Fe-based alloy Fe-Cr-Mo-W-C-Mn-Si-Zr-B powder used in the present work was not fully amorphous. During the initial stages of deposition with LENS processing, significant rapid quenching occurs and two Fe-based predominantly amorphous layers are created. With an increasing deposit thickness, new crystal phases precipitated and the microstructure coarsens due to the imposition of reheating cycles in combination with a decreasing cooling rate. The number of $\alpha$-Fe particles and other precipitated phases increases with the increasing number of deposited layers, forming graded composites with in-situ-precipitated particles in an MG matrix. The microhardness of the deposited Fe-based Fe-Cr-Mo-W-C-Mn-Si-Zr-B MGs is approximately $900 \mathrm{HV}(9.52 \mathrm{GPa})$, a value that is attractive for engineering applications.

\section{ACKNOWLEDGMENTS}

This work was sponsored by the National Science Foundation (NSF DMR00-76498). The authors thank Professor Julie M. Schoenung of the University of California, Davis, for constructive technical discussions on LENS processing.

\section{OPEN ACCESS}

This article is distributed under the terms of the Creative Commons Attribution Noncommercial License which permits any noncommercial use, distribution, and reproduction in any medium, provided the original author(s) and source are credited.

\section{REFERENCES}

1. A. Peker and W.L. Johnson: Appl. Phys. Lett., 1993, vol. 63, pp. $2342-44$.

2. A. Inoue: Mater. Trans. JIM, 1995, vol. 36, pp. 866-75.

3. W.L. Johnson: MRS Bull., 1999, vol. 24, pp. 42-56.

4. W.H. Wang, Z.X. Bao, C.X. Liu, and D.Q. Zhao: Phys. Rev. B, 2000, vol. 61, pp. 3166-69.

5. Y.H. Liu, G. Wang, R.J. Wang, D.Q. Zhao, M.X. Pan, and W.H. Wang: Science, 2007, vol. 315, pp. 1385-88.

6. J. Zhang and Y. Zhao: Nature, 2004, vol. 430, pp. 332-35.

7. D.C. Hofmann, J.-Y. Suh, A. Wiest, G. Duan, M.-L. Lind, M.D. Demetriou, and W.L. Johnson: Nature, 2008, vol. 451, pp. 1085-89.

8. Z.P. Lu, C.T. Liu, J.R. Thompson, and W.D. Porter: Phys. Rev. Lett., 2004, vol. 92, pp. 245503-245511.

9. C.T. Liu, M.F. Chisholm, and M.L. Miller: Intermetallics, 2002, vol. 10, pp. 1105-12.

10. Z.P. Lu and C.T. Liu: Phys. Rev. Lett., 2003, vol. 91, pp. $115505-$ 115511 .

11. A. Inoue, T. Masumoto, S. Arakawa, and T. Iwadachi: Mater. Trans., JIM, 1978, vol. 19, pp. 303-04.

12. A. Inoue, T. Zhang, and T. Masumoto: Mater. Trans., JIM, 1989, vol. 30, pp. $965-72$.

13. A. Inoue, T. Zhang, and T. Masumoto: J. Non-Cryst. Solids, 1993, vols. 156-158, pp. 473-80.

14. H. Habazaki, A. Kawashima, K. Asami, and K. Hashimoto: $J$. Electrochem. Soc., 1991, vol. 138, pp. 76-81.

15. K. Kobayashi, K. Hashimoto, and T. Masumoto: Res. Inst. Tohoku Univ., A, 1981, vol. 29, pp. 284-95.

16. H. Habazaki, A. Kawashima, K. Asami, and K. Hashimoto: Corros. Sci., 1992, vol. 33, pp. 225-36.

17. T.R. Anthony and H.E. Cline: J. Appl. Phys., 1978, vol. 49, pp. $1248-55$.

18. Y.-W. Kim, P.R. Strutt, and H. Nowotny: Metall. Trans. A, 1979, vol. 10A, pp. 881-86.

19. Q. Zhu, S. Qu, X. Wang, and Z. Zou: Appl. Surf. Sci., 2007, vol. 253, pp. 7060-64.

20. T.T. Wong and G.Y. Liang: Mater. Charact., 1997, vol. 38, pp. 85-89.

21. X. Wu, B. Xu, and Y. Hong: Mater. Lett., 2002, vol. 56, pp. 838-41.

22. T.M. Yue, Y.P. Su, and H.O. Yang: Mater. Lett., 2007, vol. 61, pp. 209-12.

23. C.L. Atwood, M.L. Griffith, L.D. Harwell, D.L. Greene, D.E. Reckaway, M.T. Ensz, D.M. Keicher, M.E. Schlienger, J.A. Romero, M.S. Oliver, F.P. Jeantette, and J.E. Smugeresky: Sandia Report No. SAND99-0739, Sandia National Laboratories, Albuquerque, NM, 1999, pp. 1-31.

24. B. Zheng, Y. Lin, J.E. Smugeresky, Y. Zhou, and E.J. Lavernia: TMS Lett., 2005, vol. 4, pp. 113-14.

25. M.L. Griffith, M.E. Schlienger, L.D. Harwell, M.S. Oliver, M.D. Baldwin, M.T. Ensz, M. Essien, J. Brooks, C.V. Robino, J.E Smugeresky, W.H. Hofmeister, M.J. Wert, and D.V. Nelson: Mater. Des., 1999, vol. 20, pp. 107-13. 
26. E.J. Lavernia and Y. Wu: Spray Atomization and Deposition, John Wiley \& Sons, Inc., West Sussex, United Kingdom, 1996, pp. 70 77

27. S. Annavarapu and R. Doherty: Int. J. Powder Metall., 1993 , vol. 29, pp. 331-44.

28. K.R. Cardoso, A.G. Escorial, M. Lieblich, and W.J. Botta: Mater. Sci. Eng., A, 2001, vol. 315, pp. 89-97.

29. A. Zambon, B. Badan, G. Vedovato, and E. Ramous: Mater. Sci. Eng., A, 2001, vols. 304-306, pp. 452-56.

30. Y. Liu, Z. Liu, S. Guo, Y. Du, B. Huang, J. Huang, S. Chen, and F. Liu: Intermetallics, 2005, vol. 13, pp. 393-98.

31. J.F. Ready and D.F. Farson: LIA Handbook of Laser Materials Processing: Laser Institute America, Magnolia Publishing, Orlando, FL, 2001, pp. 181-82.

32. R. Ye, J.E. Smugeresky, B. Zheng, Y. Zhou, and E.J. Lavernia: Mater. Sci. Eng., A, 2006, vol. 428, pp. 47-53.

33. B. Zheng, Y. Zhou, J.E. Smugeresky, J.M. Schoenung, and E.J. Lavernia: Metall. Mater. Trans. A, 2008, vol. 39A, pp. 222836.
34. A. Basu, A.N. Samant, S.P. Harimkar, J.D. Majumdar, I. Manna, and N.B. Dahotre: Surf. Coat. Technol., 2008, vol. 202, pp. 2623-31.

35. W. Hofmeister, M. Wert, J.E. Smugeresky, J.A. Philliber, M. Griffith, and M. Ensz: JOM-e, available online at:http://www. tms.org/pubs/journals/JOM/9907/Hofmeister/Hofmeister-9907. html, 1999, vol. 51.

36. B. Zheng, L. Ajdelsztajn, J.M. Schoenung, and E.J. Lavernia: 4th ISEC, St. Paul, MN, 2005, p. 9.

37. T. Egami: Mater. Trans., JIM, 2002, vol. 43, pp. 510-17.

38. eFunda: http://www.efunda.com/materials/alloys/alloy_home/steels_ properties.cfm.

39. Z.J. Yan, J.F. Li, S.R. He, and Y.H. Zhou: Mater. Res. Bull., 2003, vol. 38, pp. 681-89.

40. W.H. Wang: Prog. Mater. Sci., 2007, vol. 52, pp. 540-52.

41. B. Zheng: Ph.D. Dissertation, University of California, Davis, CA, 2006, pp. 242-43.

42. M.L. Lee, Y. Li, and C.A. Schuh: Acta Mater., 2004, vol. 52, pp. 4121-31.

43. J. Beuth and N. Klingbeil: JOM, 2001, vol. 53, pp. 36-39. 